Wathelet, M., D. Jongmans, M. Ohrnberger, and S. Bonnefoy-Claudet (2008). Array performances for ambient vibrations on a shallow structure and consequences over Vs inversion. Journal of Seismology, 12, 1-19.

\title{
Array performances for ambient vibrations on a shallow structure and consequences over $V_{s}$ inversion
}

\author{
Marc Wathelet ${ }^{1}$, Denis Jongmans ${ }^{1}$, Matthias Ohrnberger ${ }^{2}$, Sylvette \\ Bonnefoy-Claudet ${ }^{1,3}$
}

1) LGIT, Université Joseph Fourier, BP 53, 38041 Grenoble Cedex 9, France

2) Institut für Geowissenschaften der Universität Potsdam, POB 601553, D-14415 Potsdam, Germany

3) Institute of Radiological protection and Nuclear Safety, BP 17,92262 Fontenay aux Roses Cedex, France

Corresponding author: Marc Wathelet (marc.wathelet@ujf-grenoble.fr) 


\section{Abstract}

Valuable information about one-dimensional soil structures can be obtained by recording ambient vibrations at the surface, in which the energy contribution of surface waves predominates over the one of other types of waves. The dispersion characteristics of surface waves allow the retrieval of the shear-wave velocity as a function of depth. Microtremor studies are usually divided in two stages: deriving the dispersion (or autocorrelation) curve from the recorded signals and inverting it to obtain the site velocity profile. The possibility to determine the dispersion curve over the adequate frequency range at one site depends on the array aperture and on the wavefield spectra amplitude which can be altered by filtering effects due to the ground structure. Microtremors are usually recorded with several arrays of various apertures in order to get the spectral curves over a wide frequency band, and different methods also exist for processing the raw signals. With the objective of defining a strategy to achieve reliable results for microtremor on a shallow structure, we analyse synthetic ambient vibrations (vertical component) simulated with 333 broad-band sources for a $25 \mathrm{~m}$ deep soil layer overlying a bedrock. The first part of our study is focused on the determination of the reliable frequency range of the spectral curves (dispersion or autocorrelation) for a given array geometry. We find that the wave number limits deduced from the theoretical array response are good estimates of the valid spectral curve range. In the second part, the spectral curves are calculated with the three most popular noise-processing techniques (frequency-wavenumber, high resolution frequency-wavenumber and spatial auto-correlation methods) and inverted individually in each case. The inversions are performed with a tool based on the neighbourhood algorithm which offers a better estimation of the global uncertainties than classical linearized methods, especially if the solution is not unique. Several array apertures are necessary to construct the dispersion (autocorrelation) curves in the appropriate frequency range. Considering the final velocity profiles, the three tested methods are almost equivalent and no significant advantage was found for one particular method. With the chosen model, all methods exhibit a penetration limited to the bedrock depth, as a consequence of the filtering effect of the ground structure on the vertical component, which was observed in numerous shallow sites.

\section{Key words:}

Frequency-wavenumber, auto-correlation, inversion, surface waves, ambient vibrations, neighbourhood algorithm 


\section{Introduction}

Recording microtremors with an array of sensors is an attractive tool for in-situ measurements of shear-wave velocity $\left(V_{s}\right)$ (Asten and Henstridge [1984]). This success among the scientific community comes from the easiness to use passive sources as well as from the possibility of investigating deep sedimentary structures at a very low cost compared to crosshole or downhole techniques (Satoh et al. [2001b]). The applications of this method are not limited to earthquake engineering but may also extend to general soil characterisation. The main assumptions behind noise-array analysis are that ambient vibrations are mostly composed of surface waves and that the ground structure is approximately horizontally stratified (Tokimatsu [1997]). In such an one-dimensional heterogeneous media, surface waves are dispersive and show a variation of the apparent velocity with the frequency. Love $\left(S_{H}\right)$ and Rayleigh $\left(P-S_{V}\right)$ modes co-exist on horizontal components whilst vertical components are only affected by Rayleigh-surface waves. The majority of ambient vibration studies focuses only on vertical components and on the Rayleigh modes (i.e. Satoh et al. [2001b], Wathelet et al. [2004], Picozzi et al. [2005], Kind et al. [2005]), although some attempts were made to use Love waves as well (e.g. Chouet et al. [1998], Okada [2003], Köhler et al. [2007]). Data processing for obtaining $V_{s}$ profile from noise array measurements is divided into two main steps: deriving a spectral curve characteristic of the propagating waves (namely, a dispersion curve or auto-correlation curves), and inverting this curve to retrieve the soil structure. In such methods, the resolution at depth is intrinsically linked to the wavefield spectral amplitudes, as well as to the capabilities of the array of sensors.

Three approaches are of common use for analysing signals: the frequency wavenumber method (Lacoss et al. [1969], Horike [1985]), the high resolution frequency wavenumber method (Capon [1969], Asten and Henstridge [1984]) and the spatial auto-correlation technique (Aki [1957], Ohori et al. [2002], Roberts and Asten [2004]). Frequency-wavenumber (f-k) analysis assumes plane waves to travel across the array of sensors laid out at the surface. Considering a wave with frequency $f$, a direction of propagation and a velocity (or equivalently $k_{x}$ and $k_{y}$, wavenumbers along $\mathrm{X}$ and $\mathrm{Y}$ horizontal axis, respectively) the relative arrival times are calculated at all sensor locations and the phases are shifted according to the time delays. The array output is calculated by the summation of shifted signals in the frequency domain. If the waves travel with a given direction and velocity, all contributions will stack constructively, resulting in a high array output (usually called the beam power, Capon [1969]). The location of the maximum of the beam power in the plane $\left(k_{x}, k_{y}\right)$ provides an estimate of the velocity and of the azimuth of the travelling waves across the array. With the aim of improving the f-k method, Capon [1969] added weighting factors to each sensor contribution in the computation of the array output. They are calculated in order to minimize the energy carried by wavenumbers differing from the considered one. The high resolution frequency-wavenumber technique is theoretically able to distinguish two waves travelling at close wavenumbers in a better way than the f-k method (Capon [1969], Horike [1985]). Empirical results (Woods and Lintz [1973]) show that the resolving power of the high-resolution method is three to six times greater than the one of the f-k method. However, as outlined by Asten and Henstridge [1984], the performance of the method is very dependent upon signal to noise ratio and array design. Another possibility for analysing the recorded signals is the spatial auto-correlation technique which assumes the 
distribution of sources in the noise wavefield to be random in time and space. In the case of a single dispersive wave, Aki [1957] demonstrated that the auto-correlation ratios are functions of the phase velocities and of the array aperture. This propriety was used by several authors (Chouet et al. [1998], Roberts and Asten [2004]) to derive $V_{s}$ profiles in varied conditions.

The second step for obtaining $V_{s}$ profiles consists in inverting the spectral curves (dispersion curve or autocorrelation curves). The two frequency wavenumber techniques provide the apparent dispersion curve which can be inverted using a classical linearized algorithm (Tokimatsu [1997], Satoh et al. [2001b]), or direct search techniques like the neighbourhood algorithm (Wathelet et al. [2004]) and the genetic algorithms (Kind et al. [2005], Parolai et al. [2005]). Autocorrelation curves are commonly inverted in a two step process: the computation of the dispersion curve from the autocorrelation data and the inversion of the dispersion curve (Chouet et al. [1998]). Recently, Asten et al. [2004] and Wathelet et al. [2005] presented an adaptation of this inversion process to obtain the $V_{s}$ profile directly from the auto-correlation curves. A further step was made by Parolai et al. [2005] and Picozzi et al. [2005] who performed a joint inversion of phase-velocity dispersion and $\mathrm{H} / \mathrm{V}$ ratio curves using a genetic algorithm, accepting further assumptions on the wavefield structure.

Most of the authors having recently worked on noise array measurements favoured one of the three processing methods (Ohori et al. [2002], Kind et al. [2005], Okada [2003]). Quantifying the relative efficiency of the various ambient vibration methods is difficult for a real site where the "true" structure is often itself a matter of discussion. No papers are currently published with a comparison of all common microtremor methods based on synthetic signals which offers an undisputable reference for the model structure. We choose to simulate shallow structures (depths less than $100 \mathrm{~m}$ ) which are common in urban areas in Europe and were investigated during the SESAME project (Site Effects uSing AMbient Excitations, http://sesamefp5.obs.ujf-grenoble.fr).

The originality of this paper is to test and compare the ability of the three noise-array techniques to derive the $V_{s}$ profile on a synthetic simple case (a shallow single layer overlying a half-space) with simulated ambient vibrations. The three methods are systematically applied to three distinct array geometries with varying apertures. The same direct search technique, the neighbourhood algorithm (Sambridge [1999]), is used for inverting both dispersion and autocorrelation curves, allowing a comparison of the results. Whereas empirical relationships are often used for common array layouts (e.g. Tokimatsu [1997]) we propose objective criteria for selecting the spectral curve ranges appropriate for inversion, defined from the theoretical array response. The validity of these criteria is checked on the synthetic case.

\section{Spectral characteristics of microtremors}

Array records of microtremors are increasingly used to obtain the $V_{s}$ structure of both shallow (i.e. lower than $100 \mathrm{~m}$ depth, e.g. Wathelet [2005], Di Giulio et al. [2006]) and deep sedimentary layers (e.g., Satoh et al. [2001b,a], Scherbaum et al. [2003], Okada [2003]). Apart from the limitation resulting from the array aperture, several authors found a low-frequency limit in the determination of dispersion curves from vertical components. In the Tokachi Plain (Hokkaido, Japan) where the resonance frequency varies from 0.17 to $0.34 \mathrm{~Hz}$, Okada [2003] obtained 
dispersion curves down to $0.25 \mathrm{~Hz}$ with a maximum array aperture around $1 \mathrm{~km}$. He noticed that dispersion curves would need to be estimated below $0.2 \mathrm{~Hz}$ in order to obtain the bedrock velocity with microtremors. In Melbourne (Australia), Roberts and Asten [2004] achieved a minimum frequency of $2.5 \mathrm{~Hz}$, while the fundamental resonance frequency was estimated to $2.3 \mathrm{~Hz}$. In the Cologne area (Germany), Scherbaum et al. [2003] were able to determine stable dispersion curves on several sites down to $0.7 \mathrm{~Hz}$, which is close to the resonance frequency of the area. Later, at one of these sites, Picozzi et al. [2005] found that the impedance contrast at the sediment-bedrock interface can only be retrieved if the $\mathrm{H} / \mathrm{V}$ ratio curve is jointly inverted with the dispersion curve of Rayleigh wave. During the SESAME project (deliverable no. D06.05, http://sesame-fp5.obs.ujf-grenoble.fr//SES_TechnicalDoc.htm), various shallow sites (10 to $100 \mathrm{~m}$ of sediments over a bedrock) were investigated with microtremor arrays using 5-sec sensors. The Colfiorito Basin (Italy) is a small-size alluvial structure with a resonance frequency of about $0.9 \mathrm{~Hz}$ in its central part. On this site, Di Giulio et al. [2006] derived dispersion curves down to $0.8 \mathrm{~Hz}$, due to the drastic drop of energy by a factor 10 between 2 and $1 \mathrm{~Hz}$ on the vertical component(Figure 1(a) and (b)). A similar decrease occurs on the horizontal components but at lower frequency (between 0.7 and $0.4 \mathrm{~Hz}$ ). In the alluvial plain of the city of Liège (Belgium), a trough of $4.5 \mathrm{~Hz}$ wide was observed in the Fourier spectra of the vertical component (Figure 1(c)), just below the resonance frequency $(5 \mathrm{~Hz}$, Figure 1(d)). All these examples illustrate the difficulty to obtain the part of the dispersion curve just below the resonance frequency of a site, even with large arrays, due to the lack of coherency of propagating waves. Using an oblique impulsive shot as a source, Scherbaum et al. [2003] numerically showed that the Rayleigh wave dispersion curve is mostly excited at frequencies higher than the resonance frequency of the layered medium which acts as a highpass filter. That does not mean that it is impossible to obtain the dispersion curve at all frequencies lower than the resonance frequency, as shown by the works of Satoh et al. [2001b,a] who succeeded in estimating the S-wave velocity structure of deep basins in Taiwan and Japan, with phase velocities down to $0.2 \mathrm{~Hz}$ in favourable cases. In the Taichung basin (Taiwan, Satoh et al. [2001b]), low Fourier amplitudes between 1 and $2 \mathrm{~Hz}$ at two sites prevented to compute the phase velocities in this frequency range. The large spectral amplitudes at lower frequency allowed the phase velocities to be estimated between 0.2 and $1 \mathrm{~Hz}$. These results illustrate the complexity of deriving S-wave velocity models from spectral curve inversion, which depends on the array aperture, the filter effect of the medium and the wavefield excitation strength. For shallow structures, numerous authors have faced a lack of coherency in the wave propagation for frequencies just below the resonance frequency. This paper is focused on the numerical simulation of $1 \mathrm{D}$ shallow structures in the range $0.2-15 \mathrm{~Hz}$, for which a wide trough is observed in the Fourier spectra of the vertical component below the resonance frequency.

\section{Array capabilities}

The performance of an array for deriving phase velocity values in a wavenumber or frequency range depends on its geometry and on the wave field characteristics. At the present time there is no global agreement about the capabilities of an array designed at recording ambient vibrations. Asten and Henstridge [1984] recommended that the array diameter should be at least as large 
as the longest wavelength of interest and that the station spacing for any direction should be less than half the shortest wavelength of interest so as to avoid aliaising in the wavenumber domain. Tokimatsu [1997] proposed the following relationships between the minimum and maximum sensor spacing $\left(D_{\min }\right.$ and $\left.D_{\max }\right)$ and the minimum and maximum wavelengths $\left(\lambda_{\min }\right.$ and $\left.\lambda_{\max }\right)$ necessary to achieve reasonable results.

$$
\begin{aligned}
\lambda_{\max } & <3 D_{\max } \\
\lambda_{\min } & >2 D_{\min }
\end{aligned}
$$

The first relation comes from active source methods for linear arrays and it allows reasonable results to be obtained for ambient vibration arrays as well (Tokimatsu [1997]). The second one is derived theoretically from Nyquist wavenumber. Considering a penetration of the order of half $\lambda_{\max }$ (Park and Xia [1999]) for surface waves, the maximum depth for which $V_{s}$ can be computed is about $1.5 D_{\max }$. Accordingly, Satoh et al. [2001b] proposed the maximum wavelength to be two to four times the maximum sensor separation. Gaffet [1998] stressed out that the $\lambda_{\text {min }}$ limit obtained from the minimum sensor spacing is not well adapted to an irregular array grid, as a minimum of 2 points per wavelength is not guaranteed over the entire array. More recently, Kind et al. [2005] used the common rules of thumb to quantify the low frequency limit of the deduced dispersion curve, and a manual interpretation to identify the aliasing limits. After Woods and Lintz [1973], the resolving power of an array depends not only on the diameter of the array but also on the spatial distribution of the sensors and on the correlation between the events to be resolved. They proposed to estimate this resolving power by using the theoretical array response function. This latter function, which we also use for defining two wavenumber limits $k_{\min }$ and $k_{\max }$, is given in the $\left(k_{x}, k_{y}\right)$ plane by (Woods and Lintz [1973], Asten and Henstridge [1984]):

$$
R_{t h}\left(k_{x}, k_{y}\right)=\frac{1}{n^{2}}\left|\sum_{i=1}^{n} e^{-j\left(k_{x} x_{i}+k_{y} y_{i}\right)}\right|^{2}
$$

where $\mathrm{n}$ is the number of sensors in the array, and $\left(x_{i}, y_{i}\right)$ are their coordinates. $R_{t h}$ is computed in Figure 2(b), (e) and (h) for various array geometries (Figure 2(a), (d) and (g), respectivelly) used in the next sections. Sections are made across each of them along several azimuths and they are plotted by gray curves in Figure 2(c), (f) and (i).

For one single plane wave $S_{i}(f)=A(f) e^{j\left(x_{i} k_{x}^{(1)}+y_{i} k_{y}^{(1)}-2 \pi f t+\phi\right)}$ crossing the array at wavenumber $\left(k_{x}^{(1)}, k_{y}^{(1)}\right)$, recorded at sensor $i$, and with a phase $\phi$, the array output is

$$
R\left(k_{x}, k_{y}, f\right)=\left|\sum_{i=1}^{n} S_{i}(f) e^{-j\left(k_{x} x_{i}+k_{y} y_{i}\right)}\right|^{2}=n^{2} A^{2}(f) R_{t h}\left(k_{x}-k_{x}^{(1)}, k_{y}-k_{y}^{(1)}\right)
$$

where $A(f)$ is the amplitude spectrum. The array output is equal to the theoretical response (equation 3) translated by vector $\left(k_{x}^{(1)}, k_{y}^{(1)}\right)$ and multiplied by the square of the amplitude.

For multiple plane waves travelling across the array, $S^{(1)}$ to $S^{(m)}$, the array output is

$$
R\left(k_{x}, k_{y}, f\right)=\left|\sum_{i=1}^{n}\left(\sum_{l=1}^{m} S_{i}^{(l)}(f)\right) e^{-j\left(k_{x} x_{i}+k_{y} y_{i}\right)}\right|^{2} \leq n^{2} \sum_{l=1}^{m} R^{(l)}\left(k_{x}, k_{y}, f\right)
$$


where $R^{(l)}$ is the array output for the single plane wave $l$ defined by equation (4), and $S_{i}^{(l)}$ the wave $l$ recorded at station $i$. In this case, the array output is always lower than the sum of translated theoretical responses, and it cannot be simply interpretated as the summation of the individual shifted theoretical array responses.

From equation (3), the theoretical response $R_{t h}$ always exhibits a central peak which value is one $\left(k_{x}\right.$ and $\left.k_{y}=0\right)$ and secondary aliasing peaks which amplitude are less or equal to one. Equation (4) shows that the position of the highest peak of the real array output is directly linked to the apparent velocity and the azimuth of the propagating wave. For a simple wavefield (equation (4)), aliasing occurs for all wavenumbers greater than half the wavenumber of lateral peaks of $R_{t h}$ which reach a value of 1 . For a complex wavefield (equation (5)), aliasing is likely to occur at wavenumbers lower than this value, due to the summation of secondary peaks of $R_{t h}$. Concerning the resolving power, the thinner is the central peak of $R_{t h}$, the more capable is the array to distinguish two waves travelling at close wavenumbers (Asten and Henstridge [1984]). Resolution and aliasing limits are then directly derived from the $R_{t h}$ map. Following Woods and Lintz [1973], the resolution limit $\left(k_{\min } / 2\right)$ is taken as the radius of the central peak of $R_{t h}$ measured at the mid-height (0.5). We define the aliasing limit $\left(k_{\max }\right)$ as the lowest $\mathrm{k}$ value (greater than $k_{\min } / 2$ ) obtained at the intersection of $R_{t h}$ with the horizontal line at 0.5 , considering all directions. These two limits are illustrated in Figure 2 (b), (e), (h) and (k) by black circles while the bold black curves in Figure 2 (c), (f), (i) and (l) correspond to the azimuths with the most restrictive limit. For simple and regular array geometries, $k_{\text {min }} / 2$ and $k_{\max }$ can be linked to the maximum and minimum distances between sensors. For irregular and more usual arrays, these limits are dependent on the spatial distribution of sensors and can be more rigorously defined from the theoretical array response. In the following sections, we will check the validity of the wavenumber limits deduced from the theoretical array response on ambient vibration synthetic data for the three processing techniques.

\section{Simulated synthetic case}

The synthetic ground model is composed of a soil layer with a thickness of $25 \mathrm{~m}$ overlying an infinite bedrock. The properties of the two layers are specified in Table 1. The theoretical Rayleigh dispersion curves (the fundamental and the first four higher modes in the case of an elastic media) for this model are shown in Figure 3(a) between 1 and $15 \mathrm{~Hz}$. For frequencies below $15 \mathrm{~Hz}$, only the first five modes exist and they appear to be well separated in the velocity-frequency plane. Figure 3(b) shows the fundamental Rayleigh ellipticity curve and the $S_{H}$ transfer function. The fundamental resonance frequency is $2 \mathrm{~Hz}$ while the peak of the fundamental ellipticity is at $1.9 \mathrm{~Hz}$. This little frequency difference, recently studied by Malischewsky and Scherbaum [2004], is mainly influenced by the magnitude of the velocity contrast between the sediments and the bedrock.

On this model we set up four arrays (labelled A, B, C and D), the geometries of which are plotted in Figure 2(a), (d), (g) and (j), respectively. Array A is composed of nine sensors roughly distributed around a central sensor, with an approximate aperture of 25 meters. Array $\mathrm{B}$ is made of three triangles approximately rotated by $120^{\circ}$ and with increasing aperture up to 90 meters. Finally, arrays $\mathrm{C}$ and $\mathrm{D}$ are made of nine sensors roughly distributed around a 
central sensor, with an approximate aperture of 100 and 250 meters, respectively. Array D, the aperture of which is ten times the bedrock depth, has been designed for showing the influence of the site filtering. From the empirical rules mentioned in the section "Array capabilities", the three other arrays should be able to estimate the S-wave velocity and depth of the bedrock, and only the results of arrays $\mathrm{A}, \mathrm{B}$ and $\mathrm{C}$ will be used for the inversion. Theoretical $\mathrm{f}-\mathrm{k}$ responses for arrays A, B, C and D are shown in Figure 2(b), (e), (h) and (k), respectively. The resolution and aliasing limits defined in the previous section are summarized in Table 2. The expected maximum wavelengths deduced from the theoretical array response (resolution limit, $k_{\min } / 2$ ) and from Tokimatsu's criterion (equations 1) are compared in the last two columns of Table 2. For circular shaped arrays (A,C and D), $\lambda_{\max }$ values deduced from the theoretical array response are $13 \%$ lower than the ones estimated by Tokimatsu's criterion, while the difference reaches $53 \%$ for a non circular array like array B. The former limits are thus more conservative than the empirical rules.

Synthetic ambient vibrations were computed during 6 minutes using the method proposed by Hisada [1994, 1995] and used by Bonnefoy-Claudet et al. [2006], which is valid for a onedimensional model with sources and receivers placed at any depth. A first dataset was generated for arrays A to C with 333 source points randomly distributed between 140 and $750 \mathrm{~m}$ from the array centers (Figure 4(a)). A second dataset with the same number of source points was also generated for array D from 250 to $1500 \mathrm{~m}$. Sources are punctual forces with delta-like functions of random amplitudes and directions, and with a frequency spectrum from 0 to $15 \mathrm{~Hz}$, showing a flat part between 0.15 to $3 \mathrm{~Hz}$ (Figure 4(b)). The spectrum is limited to $15 \mathrm{~Hz}$ in order to reduce $\mathrm{CPU}$ time. All types of waves are modelled, generating a complete wave field containing body, Love and Rayleigh waves. Previous studies (e.g. SESAME Project, http://sesamefp5.obs.ujf-grenoble.fr/Delivrables/Del-D24-Wp13.pdf) pointed out that the approximation of plane waves is valid for a ratio $\mathrm{d} / \mathrm{r}$ over 2.5, where $d$ is the distance between the array center and the sources, and $r$ is array radius (half of the aperture). Figure 4(c) shows, for the four arrays $\mathrm{A}, \mathrm{B}, \mathrm{C}$ and $\mathrm{D}$, the cumulative distribution of $d / r$ ratios. It turned out that less than $1 \%$ (arrays $\mathrm{B}$ and $\mathrm{C}$ ) and $3 \%$ (array D) of the sources did not match this requirement.

The spectra of the vertical $(\mathrm{V})$ and one horizontal $(\mathrm{H})$ component of the central station are shown in Figure $4(\mathrm{~d})$ and (e), as well as the $\mathrm{H} / \mathrm{V}$ ratio (Figure $4(\mathrm{f})$ ). The computation is performed over a series of short time windows (e.g. from 10 to 50 cycles at a given frequency) and statistics are obtained for all spectra (Koller et al. [2004]). The Fourier spectra show that the energy of the vertical component vanishes in the vicinity of and below the fundamental frequency $(1.9 \mathrm{~Hz})$, as reported in previous sections, while the energy on the horizontal component decreases below $1.5 \mathrm{~Hz}$. This high-pass filtering effect is particularly severe in this two-layer structure where all the energy which is not trapped in the upper layer flows into the infinite half-space. The frequency of the $\mathrm{H} / \mathrm{V}$ peak $(2 \mathrm{~Hz})$ matches the resonance frequency of the soft layer (Figure 3(b)).

\section{Frequency-wavenumber method}

The contributions of 333 sources are summed together to simulate ambient vibrations. To estimate the uncertainty on the apparent velocity determination, the signals are split in several 
short time windows for which the beam power are computed. For each time window, the velocity of the beam power peak is searched for wavenumbers below $1.5 \mathrm{rad} / \mathrm{m}$ and for velocities between 150 and $2000 \mathrm{~m} / \mathrm{s}$. From a coarse griding in the wavenumber plane, the vector $\left(k_{x}, k_{y}\right)$ of the highest peak is iteratively refined to an arbitrary small precision. Thus, for each frequency band, an histogram of the velocities at the observed maxima is constructed (e.g. Figure 5(a) for array $\mathrm{C}$ and 10 cycles) as proposed by Ohrnberger et al. [2004]. Slowness axis is chosen for the statistics because slowness depends linearly upon the measured time shifts. This choice affects the shape of the density probability function. The areas below the histograms are normalized to one in the slowness domain, explaining the high values for the probability density functions. The curves in Figure 5(c) are sections across the histograms of Figure 5(a) and 5(b) at $3 \mathrm{~Hz}$.

The influence of the window length is first studied for array $\mathrm{C}$ by calculating the histograms for time windows containing 10 and 50 cycles (Figure 5(a) and 5(b), respectively). The results are compared to the theoretical dispersion curves drawn in the same figure. In Figure 5(a), the velocity average deviates from the theoretical dispersion curve with a constant bias of 50 or $100 \mathrm{~m} / \mathrm{s}$ towards lower velocity, whereas for the 50-cycle case (Figure 5(b)), all the velocity estimates are closer to the theoretical curve and the standard deviations are much smaller. Both cases are calculated with the same duration of signals (six minutes), resulting in five times more windows in the 10-cycle case. To test the robustness of the statistics, one minute and 12 seconds of signals are also processed with time windows of 10 cycles, containing the same number of time windows as in the 50-cycle case calculated with the 6 minutes of signals. The obtained histograms are the same as in Figure 5(a). Hence, with short time windows, increasing the number of windows neither reduces the gap to the theoretical curve nor the size of resulting error bars.

A similar processing is applied to the signals of arrays A, B and D (six minutes of signals and time windows of 50 cycles). The velocity histograms of arrays A, B, C and D are compared in Figure 6(a), 6(b), 5(b), and 6(c), respectively. The proposed validity curves are drawn in the same way as in Figure 5.

For arrays $\mathrm{A}$ to $\mathrm{C}$, the phase velocity values are correctly estimated between $k_{\min }$ and $k_{\max } / 2$, except around $6 \mathrm{~Hz}$ for array A and over $3.5 \mathrm{~Hz}$ for array D. Between $k_{\min } / 2$ and $k_{\min }$, an increase of the uncertainty is observed for all arrays, associated with a deviation from the theoretical dispersion curve for arrays $\mathrm{A}$ and $\mathrm{B}$. Only in case of array $\mathrm{C}$ could the range be extended to $k_{\text {min }} / 2$ (Figure 5(b)). In Figure 5(b) (array C) and Figure 6(c) (array D), bad estimations of velocity due to aliasing take place over $k_{\max } / 2$. A similar conclusion could be drawn to a lesser extent for array B (Figure 6(b)). Due to the limited available frequency range, the aliasing effect cannot be observed for array A (Figure 6(a)).

For array A, the measured velocity is slightly over the theoretical Rayleigh velocity around $6 \mathrm{~Hz}$. If we consider a single source acting far from array A, a similar bump is observed in the dispersion curve (Figure 7(a)), while the dispersion curve is perfectly retrieved with one source from $2.5 \mathrm{~Hz}$ to $20 \mathrm{~Hz}$ for arrays B and C (not shown). Figure 7(b) and (c) shows the responses of arrays $\mathrm{A}$ and $\mathrm{B}$, respectively, in the plane $\left(k_{x}, k_{y}\right)$ for the single source at $6.5 \mathrm{~Hz}$. A single large maximum appear for array A (Figure 7(b)) whereas two individual peaks are visible for array B (Figure $7(\mathrm{c})$ ), corresponding to the slownesses of the fundamental and the first higher mode. Hence, the bad performances around $6 \mathrm{~Hz}$, within the array limits deduced from the 
theoretical array response, is due to the presence of two dominant modes in the wavefield, that array A is not able to resolve.

For the largest array D, a strong deviation from the correct phase velocity is observed below $2.2 \mathrm{~Hz}$, which is also the lower limit of the dispersion curve for the single source analysis with array A (Figure 7(a)). These results highlight the lack of energy below the resonance frequency, resulting from the filtering of the layer. As explained before, the dispersion curve of array D is not considered during the inversion.

For each array (except array D), an average and a standard deviation is calculated between $k_{\min }$ and $k_{\max } / 2$ based on the histograms of Figure $5(\mathrm{~b})$ and 6 . The three curves are averaged taking into account the respective weights (number of time windows) to construct the final dispersion curve plotted in Figure 11 (b). The measured dispersion is reliable for frequencies above $3 \mathrm{~Hz}$. This limit is linked to the decrease of the Rayleigh wave energy (vertical component) at and below the fundamental resonance frequency $(2 \mathrm{~Hz})$.

The obtained dispersion curve is inverted with five distinct runs of the neighbourhood algorithm, generating a total of 50,000 models. The parameterised model consists of a sediment layer whose wave velocity increases with depth according to a power law, and a half-space at the base. The gradient introduces a supplementary degree of freedom for scanning more models, compared to a uniform sediment layer over a half space. The parameters to invert are six: $V_{p}$ and $V_{s}$ in the two layers, the layer thickness and the velocity increase between the top and the bottom of the sediment layer. Contrary to a classical approach, $V_{p}$ is kept as a parameter to be sure that all possible value of $V_{s}$ are investigated. Figure 11(a) shows the $V_{s}$ profile for all models fitting the dispersion curve with a misfit less than one. The misfit function is defined by the following equation (Wathelet et al. [2004]),

$$
\text { misfit }=\sqrt{\sum_{i=1}^{n_{F}} \frac{\left(x_{d i}-x_{c i}\right)^{2}}{\sigma_{i}^{2} n_{F}}}
$$

where, $x_{d i}$ is the velocity of data curve at frequency $f_{i}, x_{c i}$ is the velocity of calculated curve at frequency $f_{i}, \sigma_{i}$ is the uncertainty of the frequency samples considered, and $n_{F}$ is the number of frequency samples. The dispersion curves corresponding to the misfit threshold of one are plotted in Figure 11(c). Dispersion curve inversion leads to a good definition of the $V_{s}$ profile for the first $25 \mathrm{~m}$. Below this depth, a large range of $V_{s}$ values may explain the measured dispersion curve, due to the lack of information at low frequency.

\section{High resolution method}

For the four arrays A, B, C, and D, the dispersion curves were calculated by searching the maximum of the high-resolution frequency wavenumber estimator defined by Capon [1969]. The estimator depends upon the cross spectral matrix calculated with frequency averaging and inverted after an eigen value decomposition. The process is performed for sliding time windows (50 cycles) during the 6 minutes of available signals. The results are shown in Figure 8(a) to $8(\mathrm{~d})$, for arrays $\mathrm{A}, \mathrm{B}, \mathrm{C}$ and $\mathrm{D}$, respectively, as well as the four validity curves used for analysing the results of the f-k method. From figure 8, the velocity estimation of arrays B and 
$\mathrm{C}$ is reliable between $k_{\min }$ and $k_{\max } / 2$, similarly to the results of the $\mathrm{f}-\mathrm{k}$ method. For arrays $\mathrm{B}$ and $\mathrm{C}$, the high resolution method still provides acceptable results below $k_{\min } / 2$ (down to around $k_{\min } / 3$ in both cases) and, extending the validity range at low frequency with, however, a regular increase of the uncertainty. On the contrary, no improvement is provided by the high resolution method at high frequency, as shown by the dramatic increase of error bars over $k_{\max } / 2$. For array $\mathrm{A}$, the method does not succeed better than the $\mathrm{f}-\mathrm{k}$ method and also overestimates the velocity between 6 and $9 \mathrm{~Hz}$. For array D, no improvements are obtained for frequencies below $2.2 \mathrm{~Hz}$, confirming that the lack of resolution power does not come from the array geometry but from the lack of energy in the wavefield (Figure 4(d)).

After selecting the velocity values between the validity curves $\left(k_{\min } / 2\right.$ and $\left.k_{\max } / 2\right)$, an average dispersion curve is calculated to feed the inversion algorithm. We performed exactly the same inversion processes as for the f-k results (Figure 11 (c) and (d)). The $V_{s}$ value is accurately retrieved down to the major impedance contrast but $V_{s}$ below $25 \mathrm{~m}$ is not constrained from the dispersion curve. In this case, the extension of the curve in the low frequency range does not induce a significant improvement in the inverted $V_{s}$ profile.

\section{Spatial autocorrelation}

Finally, the signals simulated for the three arrays A, B and C were analysed using the spatial auto-correlation method described in Wathelet [2005]. The azimuths and the distances between all couples of stations are shown in Figure 9 (a) to (c). The pairs of gray circles are the selected rings for the spatial auto-correlation curves computation. Distances are summarized in Table 3. A total of 15 auto-correlation curves (five by array) was calculated for time windows of 25 cycles. Only one curve per array is shown in Figure 9 (d) to (f) with gray dots and gray errors bars. The consistency of all 15 auto-correlation curves is checked on dispersion curves in Figure 10(a) to 10 (c), for arrays A to C, respectively. Only the samples fitting the criteria shown in Figure 10 (see Wathelet [2005] for details) are kept for the autocorrelation curve inversion. They are plot with black dots in Figure 9 (d) to (f).

The 15 autocorrelation curves with the selected samples are inverted with five independent runs keeping the same parameterisation as for the two preceding methods. The results are shown in Figure 11. Only three autocorrelation curves among the fifteen are plotted in Figure 11(g) to 11(i). A good agreement is found between the calculated curves and the observed autocorrelations (black dots and their error bars) even below $2 \mathrm{~Hz} . V_{s}$ models deduced from the inversion (Figure $11(\mathrm{~g})$ and $(\mathrm{h})$ ) exhibit the same features as the ones obtained with the other two methods: the $V_{s}$ value is correct in the top layer while $V_{s}$ below the major impedance contrast is very poorly resolved. Comparing Figure 11(a), 11(c) and 11(e), the auto-correlation method offers a little more constraint on $V_{s}$ in the bottom layer, resulting from the additional information at low frequency. The dispersion curves corresponding to the models are drawn for comparison with the theoretical ones in Figure 11(f). The auto-correlation method correctly retrieves the dispersion curve for all frequencies above $2.5 \mathrm{~Hz}$. Below this value, the uncertainty on the curve determination dramatically increases (Figure 11(f)). 


\section{Discussion and Conclusions}

Three processing methods have been tested to retrieve the dispersion characteristics (dispersion curves or autocorrelation curves) on a two-layer model from simulated noise array measurements: the f-k method, the high-resolution method and the spectral autocorrelation technique. The vertical components of synthetic signals were processed and the dispersion and autocorrelation curves were inverted with the same technique (neighbourhood algorithm) to obtain the $V_{s}$ profile. During the first phase, a special attention was paid to the required time window length and to the reliable frequency range of the spectral curves, which depends on the array geometry and on the wavefield structure (number of simultaneously acting sources and the presence of higher modes). We propose to estimate the reliable frequency range after the computation of the theoretical array response which takes into account the real array geometry, contrary to more classical rules of thumb calculated from the minimum and maximum distances between sensors which are less conservative. Comparing the derived dispersion curves to the theoretical one, we conclude that the wave number limits deduced from the theoretical array response are consistent with the capabilities of the $\mathrm{f}-\mathrm{k}$ method and can be used as first-order objective criteria. Outside those limits, the calculated curves may exhibit strong bias. As a consequence, several array apertures have to be systematically used to construct the dispersion curves piece by piece to cover the widest frequency range. For the processed cases, a time window length of at least 50 cycles is required to obtain a low uncertainty on the dispersion curve. The high-resolution $\mathrm{f}-\mathrm{k}$ method was found to be more efficient for some arrays than the $\mathrm{f}-\mathrm{k}$ approach in defining the dispersion curve, particularly in the low frequency range. However, such improvement was not systematically observed for all arrays and appeared to be linked to an increase of the uncertainty. The comparison of the spectral curves before inversion (Figure 11(b), (d) and (g) to (i)) shows that the high-resolution $\mathrm{f}-\mathrm{k}$ and the auto-correlation methods allow information in the low frequency range to be gained (down to $2.5 \mathrm{~Hz}$ and $2 \mathrm{~Hz}$, respectively) compared to the $\mathrm{f}-\mathrm{k}$ technique $(3 \mathrm{~Hz})$. The combined application of the three techniques is then recommended to check the validity and to increase the reliability of the results. For the three methods, a lack of resolution power was observed for array A, even in the proposed validity limits, due to the excitation of the first higher mode. From the inversion point of view, all three methods have almost the same efficiency for retrieving the $V_{s}$ profile of this two-layer synthetic case, down to about $25 \mathrm{~m}$ which is the depth of the bedrock. For the shallow structure studied here, no method is able to determine the magnitude of the velocity contrast between the sediments and the bedrock, even with larger arrays. This limited penetration depth is a direct consequence of the high-pass filtering effect of the ground structure on the vertical component, which is particularly severe for a pure 2-layer medium. This phenomenon has already been frequently observed in numerous real sedimentary sites as a trough in the spectral amplitudes below the resonance frequency, which makes difficult the computation of the dispersion curve in this frequency band. The presence of these low spectral amplitudes can be a limitation for assessing the S-wave velocity in the top of the bedrock. To overcome this problem, Picozzi et al. [2005] proposed to recover S-wave velocity of the bedrock by a joint inversion of the dispersion curve and the shape of the $\mathrm{H} / \mathrm{V}$ spectra, assuming a 1:1 ratio between the horizontal and vertical loading forces. Even if such joint inversion is promising, this supplementary assumption can be 
hard to check for routine applications, and recent studies (Bard [2004]) pointed out that the shape of the $\mathrm{H} / \mathrm{V}$ ratio can be sensitive to different external conditions like wind or strong rains. Another alternative to address the problem of penetration is to consider horizontal components which are richer in low frequency waves than vertical ones.

\section{Acknowledgements}

This work was supported by the SESAME European project ("Site EffectS assessment using Ambient Excitation", Project EVG1-CT-2000-00026). We would like to thank all members of the SESAME project for all the fruitful discussions about ambient vibrations, and particularly C. Cornou. We also address our gratitude to Prof. T. Satoh and an anonymous reviewer whose comments substantially improve our original manuscript. 


\section{References}

K. Aki. Space and time spectra of stationary stochastic waves, with special reference to microtremors. Bull. Earthq. Res. Inst., 35:415-456, 1957.

M. W. Asten and J. D. Henstridge. Array estimators and use of microseisms for reconnaissance of sedimentary basins. Geophysics, 49:1828-1837, 1984.

M. W. Asten, T. DHU, and N. Lam. Optimized array design for microtremor array studies applied to site classification; comparison of results with SCPT logs. In Proc. of 13th World Conf. on Earthquake Engineering, Vancouver, B.C., Canada, August 1-6, 2004.

P.-Y. Bard. The SESAME Projet: an overview and main results. In Proc. of 13th World Conf. on Earthquake Engineering, Vancouver, B.C., Canada, August 1-6, pages paper No. 2207, 16p., 2004.

S. Bonnefoy-Claudet, C. Cornou, P.-Y. Bard, F. Cotton, and J. Kristek. H/V ratio: a tool for site effect evalutation. Results from 1D noise simulations. Geophys. J. Int., 167:827-837, 2006.

J. Capon. High-resolution frequency-wavenumber spectrum analysis. Proc. IEEE, 57:14081418, 1969.

B. Chouet, G. De Luca, G. Milana, P. Dawson, M. Martini, and R. Scarpa. Shallow velocity structure of Stromboli Volcano, Italy, derived from small-aperture array measurements of strombolian tremor. Bull. Seism. Soc. Am., 88:653-666, 1998.

G. Di Giulio, C. Cornou, M. Ohrnberger, M. Wathelet, and A. Rovelli. Deriving Wavefield Characteristics and Shear-Velocity Profiles from Two-Dimensional Small-Aperture Arrays Analysis of Ambient Vibrations in a Small-Size Alluvial Basin, Colfiorito, Italy. Bull. Seism. Soc. Am., 96:1915-1933, 2006.

S. Gaffet. A dense array experiment for the observation of waveform perturbations. Soil Dynamics and Earthquake Engineering, 17:475-484, 1998.

Y. Hisada. An efficient method for computing Green's functions for a layered half-space with sources and receivers at close depths. Bull. Seism. Soc. Am., 84:1456-1472, 1994.

Y. Hisada. An efficient method for computing Green's functions for a layered half-space with sources and receivers at close depths (Part 2). Bull. Seism. Soc. Am., 85:1080-1093, 1995.

M. Horike. Inversion of phase velocity of long-period microtremors to the S-wave-velocity structure down to the basement in urbanized areas. Journal of Physics of the Earth, 33: 59-96, 1985.

F. Kind, D. Fäh, and D. Giardini. Array measurements of S-wave velocities from ambient vibrations. Geophys. J. Int., 160:114-126, 2005. 
A. Köhler, M. Ohrnberger, F. Scherbaum, M. Wathelet, and C. Cornou. Assessing the reliability of the modified three-component spatial autocorrelation technique. Geophys. J. Int., 168: 779-796, 2007.

M. Koller, J.-L. Chatelain, B. Guillier, A.-M. Duval, K. Atakan, C. Lacave, P.-Y. Bard, and SESAME-Team. Practical user guidelines and software for the implementation of the H/V ratio technique: measuring conditions, processing method and results interpretation. In Proc. of 13th World Conf. on Earthquake Engineering, Vancouver, B.C., Canada, August 1-6, 2004.

R. T. Lacoss, E. J. Kelly, and M. N. Toksöz. Estimation of seismic noise structure using arrays. Geophysics, 34:21-38, 1969.

P. G. Malischewsky and F. Scherbaum. Love's formula and H/V-ratio (ellipticity) of Rayleigh waves. Wave Motion, 40:57-67, 2004.

M. Ohori, A Nobata, and K. Wakamatsu. A Comparison of ESAC and FK Methods of Estimating Phase Velocity Using Arbitrarily Shaped Microtremor Arrays. Bull. Seism. Soc. Am., 92:2323-2332, 2002.

M. Ohrnberger, E. Schissele, C. Cornou, S. Bonnefoy-Claudet, M. Wathelet, A. Savvaidis, F. Scherbaum, and D. Jongmans. Frequency wavenumber and spatial autocorrelation methods for dispersion curve determination from ambient vibration recordings. In Proc. of 13th World Conf. on Earthquake Engineering, Vancouver, B.C., Canada, August 1-6, 2004.

H. Okada. The Microseismic Survey Method: Society of Exploration Geophysicists of Japan. Translated by Koya Suto, Geophysical Monograph Series No. 12. Society of Exploration Geophysicists, 2003.

Miller; R. D. Park, C. B. and J. Xia. Multichannel analysis of surface waves. Geophysics, 64: 800-808, 1999.

S. Parolai, M. Picozzi, S. M. Richwalski, and C. Milkereit. Joint inversion of phase velocity dispersion and $\mathrm{H} / \mathrm{V}$ ratio curves from seismic noise recordings using a genetic algorithm, considering higher modes. Geophysical Research Letter, 32:L01303, doi:10.1029/2004GL021115, 2005.

M. Picozzi, S. Parolai, and S. M. Richwalski. Joint inversion of $\mathrm{H} / \mathrm{V}$ ratios and dispersion curves from seismic noise: Estimating the S-wave velocity of bedrock. Geophysical Research Letter, 32:L11308, doi:10.1029/2005GL022878, 2005.

J. C. Roberts and M. W. Asten. Resolving a velocity inversion at the geotechnical scale using the microtremor (passive seismic) survey method. Exploration Geophysics, 35:14-18, 2004.

M. Sambridge. Geophysical inversion with a neighbourhood algorithm: I. Searching a parameter space. Geophys. J. Int., 138:479-494, 1999. 
T. Satoh, H. Kawase, T. Iwata, S. Higashi, T. Sato, K. Irikura, and H.-C. Huang. S-Wave Velocity Structure of the Taichung Basin, Taiwan, Estimated from Array and Single-Station Records of Microtremors. Bull. Seism. Soc. Am., 91:1267-1282, 2001a.

T. Satoh, H. Kawase, and S. I. Matsushima. Estimation of S-Wave Velocity Structures in and around the Sendai Basin, Japan, Using Array Records of Microtremors. Bull. Seism. Soc. Am., 91:206-218, 2001b.

F. Scherbaum, K.-G. Hinzen, and M. Ohrnberger. Determination of shallow shear wave velocity profiles in the Cologne/Germany area using ambient vibrations. Geophys. J. Int., 152:597$612,2003$.

K. Tokimatsu. Geotechnical site characterization using surface waves. In Ishihara (ed), editor, Proc. 1st Intl. Conf. Earthquake Geotechnical Engineering, volume 3, pages 1333-1368. Balkema, 1997.

M. Wathelet. Array recordings of ambient vibrations: surface-wave inversion. PhD thesis, Université de Liège, Belgium, 2005.

M. Wathelet, D. Jongmans, and M. Ohrnberger. Surface wave inversion using a direct search algorithm and its application to ambient vibration measurements. Near Surface Geophysics, $2: 211-221,2004$.

M. Wathelet, D. Jongmans, and M. Ohrnberger. Direct inversion of Spatial Auto Correlation Curves with the Neighborhood Algorithm. Bull. Seism. Soc. Am., 95:1787-1800, 2005.

J. W. Woods and P. L. Lintz. Plane waves at small arrays. Geophysics, 38:1023-1041, 1973. 


\section{Tables}

\begin{tabular}{cccccc}
\hline Thickness & $V_{p}$ & $V_{s}$ & Density & $Q_{p}$ & $Q_{s}$ \\
\hline $25 \mathrm{~m}$ & $1350 \mathrm{~m} / \mathrm{s}$ & $200 \mathrm{~m} / \mathrm{s}$ & $1.9 \mathrm{t} / \mathrm{m}^{3}$ & 50 & 25 \\
- & $2000 \mathrm{~m} / \mathrm{s}$ & $1000 \mathrm{~m} / \mathrm{s}$ & $2.5 \mathrm{t} / \mathrm{m}^{3}$ & 100 & 50
\end{tabular}

Table 1: Properties of the synthetic model.

\begin{tabular}{c|cccccccc}
\hline Array name & Min. dist. & Max. dist. & $k_{\min } / 2$ & $k_{\max } / 2$ & $f_{\min }$ & $f_{\max }$ & $\frac{2 \pi}{k_{\min } / 2}$ & $3 D_{\max }$ \\
\hline $\mathrm{A}$ & $8 \mathrm{~m}$ & $25 \mathrm{~m}$ & 0.095 & 0.75 & 4.2 & $\geq 15.0$ & 66 & 75 \\
$\mathrm{~B}$ & $13 \mathrm{~m}$ & $87 \mathrm{~m}$ & 0.037 & 0.25 & 3.0 & 7.6 & 170 & 261 \\
$\mathrm{C}$ & $34 \mathrm{~m}$ & $99 \mathrm{~m}$ & 0.024 & 0.20 & 2.4 & 6.2 & 262 & 297 \\
$\mathrm{D}$ & $84 \mathrm{~m}$ & $248 \mathrm{~m}$ & 0.0096 & 0.075 & 1.4 & 4.0 & 655 & 744 \\
\hline
\end{tabular}

Table 2: For arays A to D, minimum and maximum distances between sensors, minimum and maximum wavenumbers deduced from the theoretical frequency-wavenumber responses in Figure 2(b), (e), (h) and (k). The next columns are the minimum and maximum frequencies read on the theoretical Rayleigh fundamental mode corresponding to $k_{\min } / 2$ and $k_{\max } / 2$, respectively. The last two columns are the expected maximum wavelength calculated from $k_{\min } / 2$ and from Tokimatsu's criterion (equations 1, Tokimatsu [1997]). 


\begin{tabular}{c|ccc}
\hline Array name & Min. radius & Max. radius & Number of pairs \\
\hline A & 7.8 & 9.4 & 9 \\
A & 12.1 & 13.2 & 9 \\
A & 15.3 & 17.0 & 9 \\
A & 21.2 & 22.5 & 9 \\
A & 24.4 & 25.3 & 9 \\
\hline B & 12.5 & 18.0 & 6 \\
B & 22.0 & 26.3 & 9 \\
B & 34.7 & 43.3 & 12 \\
B & 49.1 & 63.8 & 12 \\
B & 73.8 & 87.3 & 6 \\
\hline C & 33.5 & 35.0 & 9 \\
C & 48.4 & 54 & 9 \\
C & 63.9 & 65.1 & 9 \\
C & 85.6 & 87.3 & 9 \\
C & 97.5 & 99.4 & 9 \\
\hline
\end{tabular}

Table 3: Distance limits for the selected rings for arrays A, B and C. The last column is the number of station couples included in each ring. Distances are expressed in meters. 


\section{Figure captions}
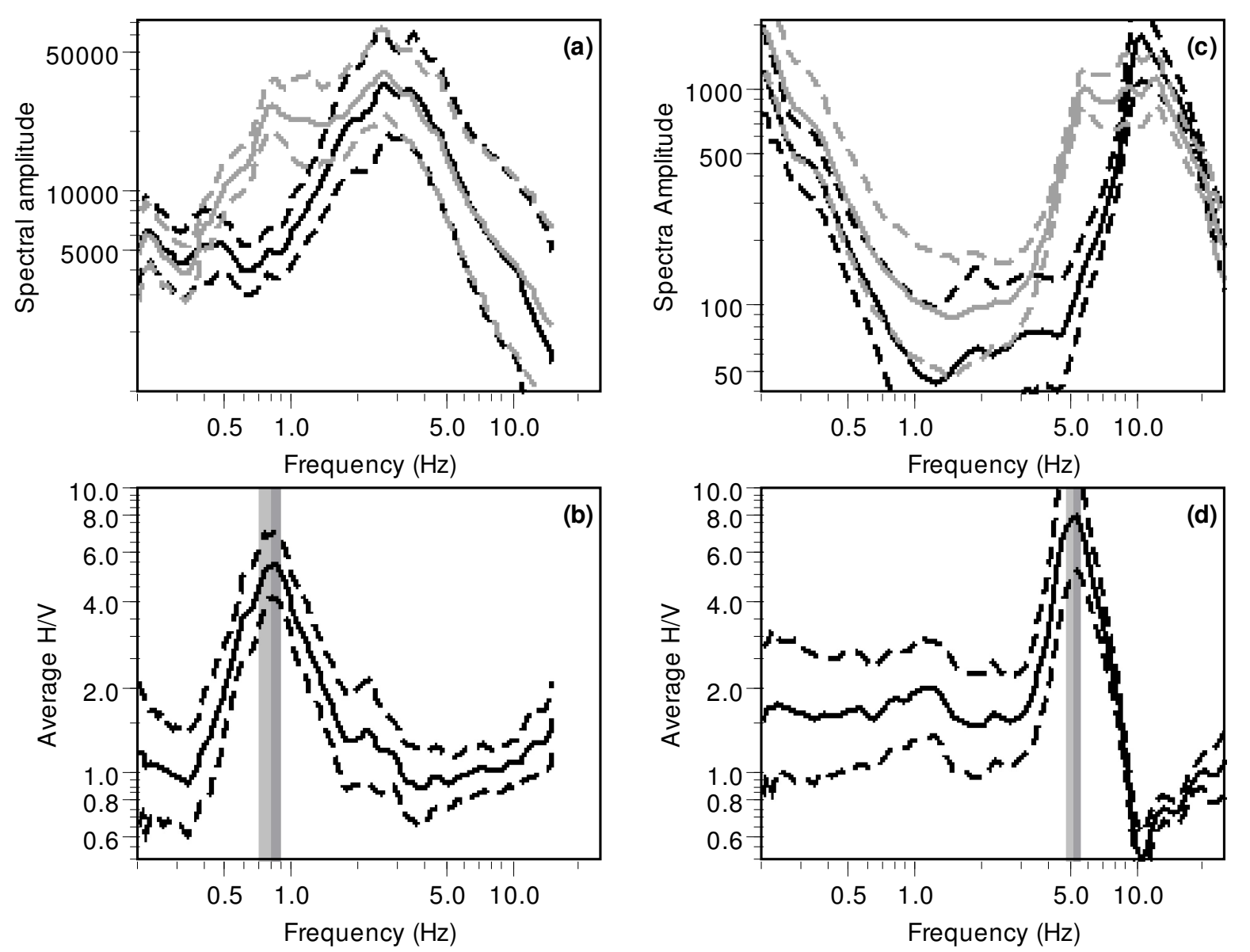

Figure 1: Top: vertical (black curves) and horizontal (gray curves) spectra measured on microtremors at two real shallow sites: (a) Colfiorito plain, Italy, and (c) Liège city, Belgium. Plain lines are average curves and dashed lines represent one standard deviation. Bottom: corresponding $\mathrm{H} / \mathrm{V}$ ratios measured at (b) Colfiorito plain and (d) at Liège city. The average and the standard deviation of the peak frequency is indicated by the grayed areas. 
Wathelet et al.
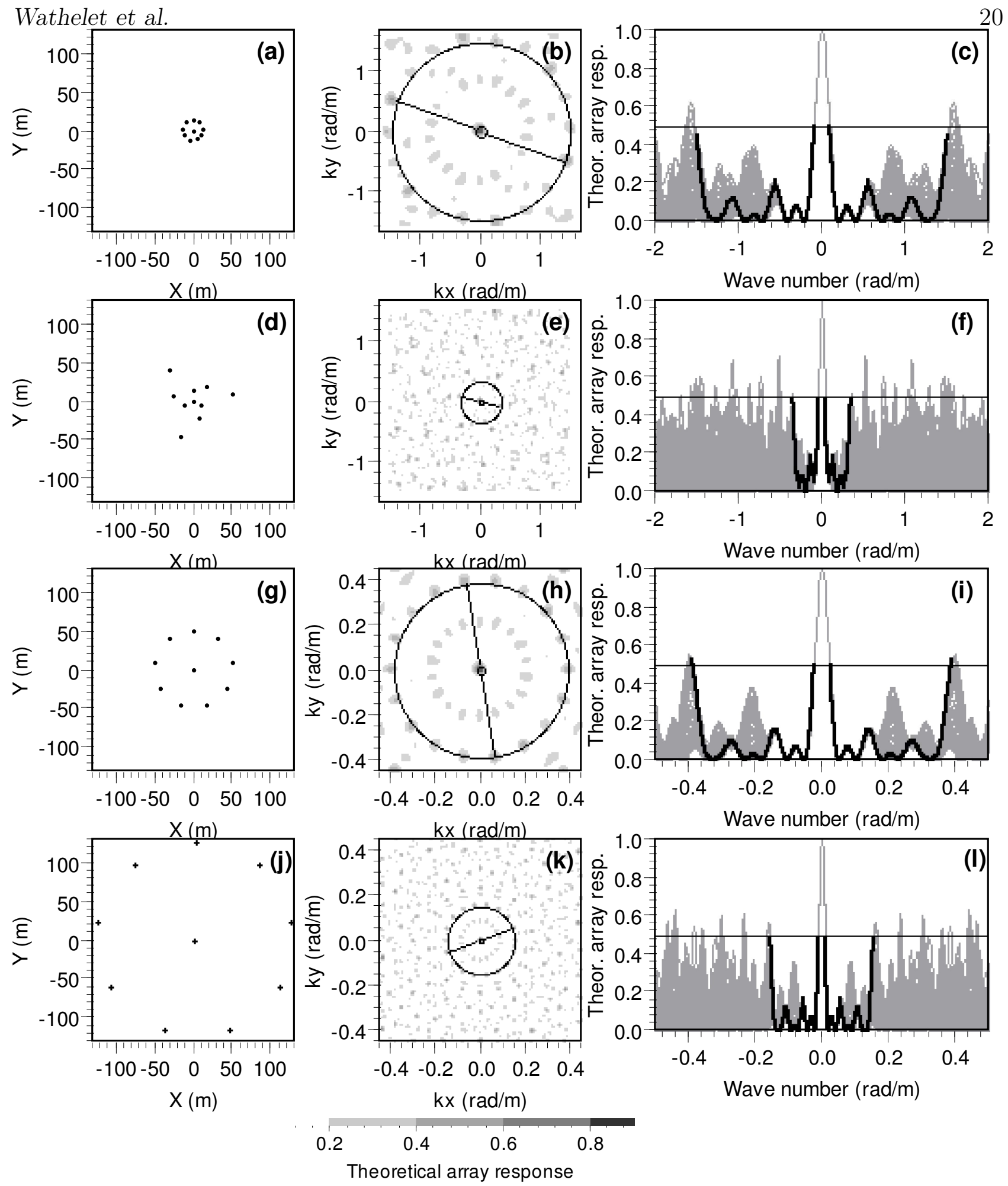

Figure 2: (a), (d), (g) and (j) Geometries of arrays A, B, C and D, respectively. (b), (e), (h) and (k) Their corresponding theoretical frequency-wavenumber responses. The circles correspond to the chosen wavenumber limits detailed in Table 2. (c), (f), (i) and (l) Sections across several azimuths for the theoretical frequency-wavenumber grids of arrays A, B, C and D, respectively. The black curve corresponds to the orientation of the line drawn in Figure (b), (e), (h) and (k). Note that the wave number scale is not the same for arrays A-B and C-D. 

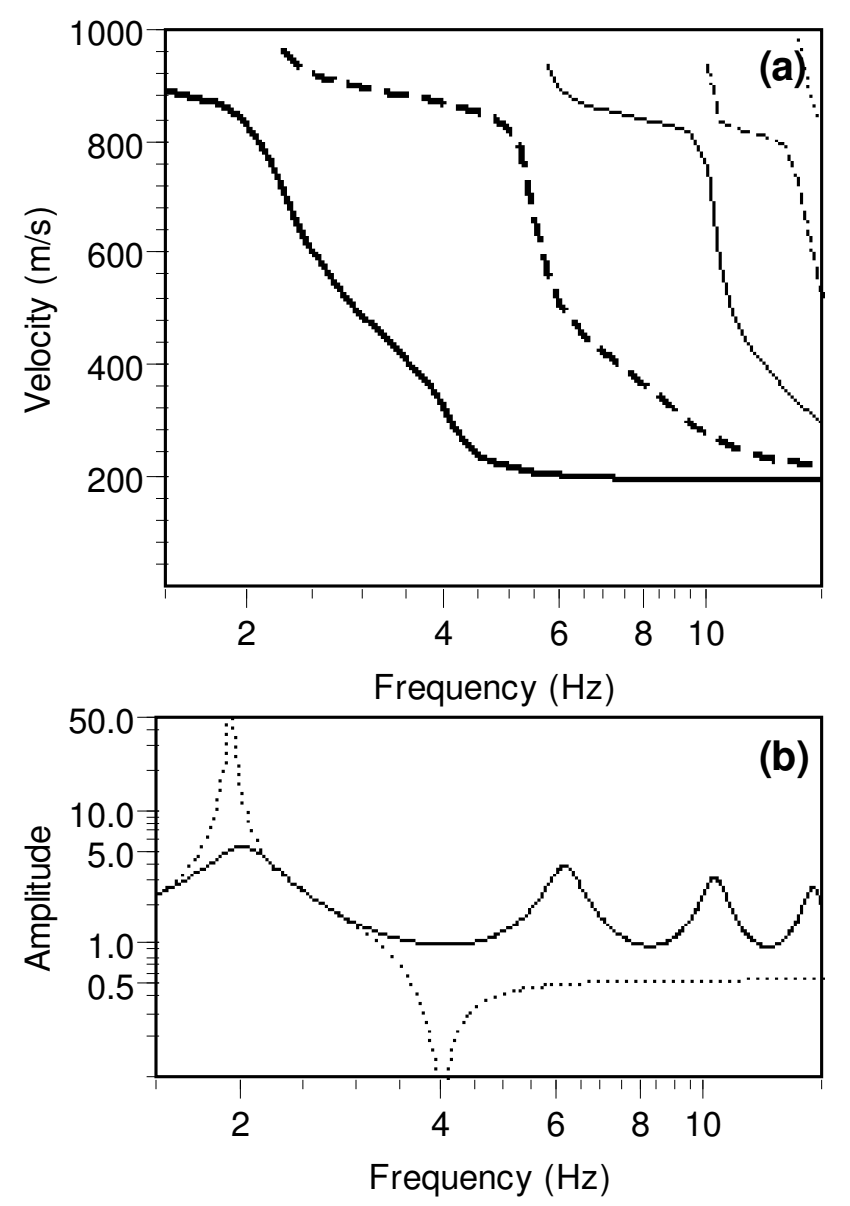

Figure 3: Synthetic model. (a) Dispersion curves for Rayleigh modes: fundamental mode (thick plain line), first (thick dashed line), second (thin plain line), third (thin dashed line), and fourth higher modes (thin dotted line). Other modes do not exist in the plotted range. (b) Fundamental Rayleigh ellipticity curve (dotted line) and theoretical $S_{H}$ transfer function for the synthetic model (plain line). 

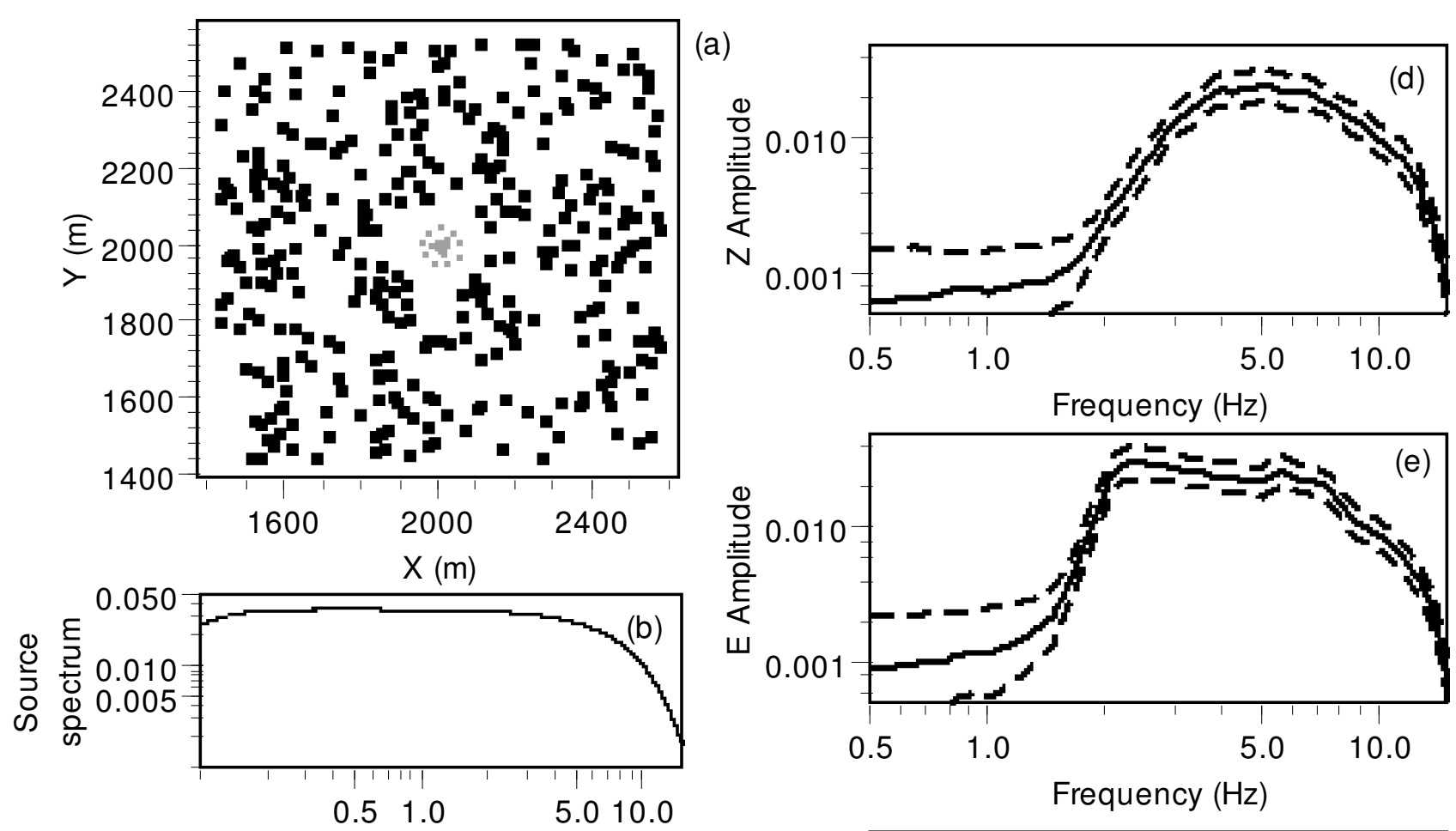

Frequency $(\mathrm{Hz})$
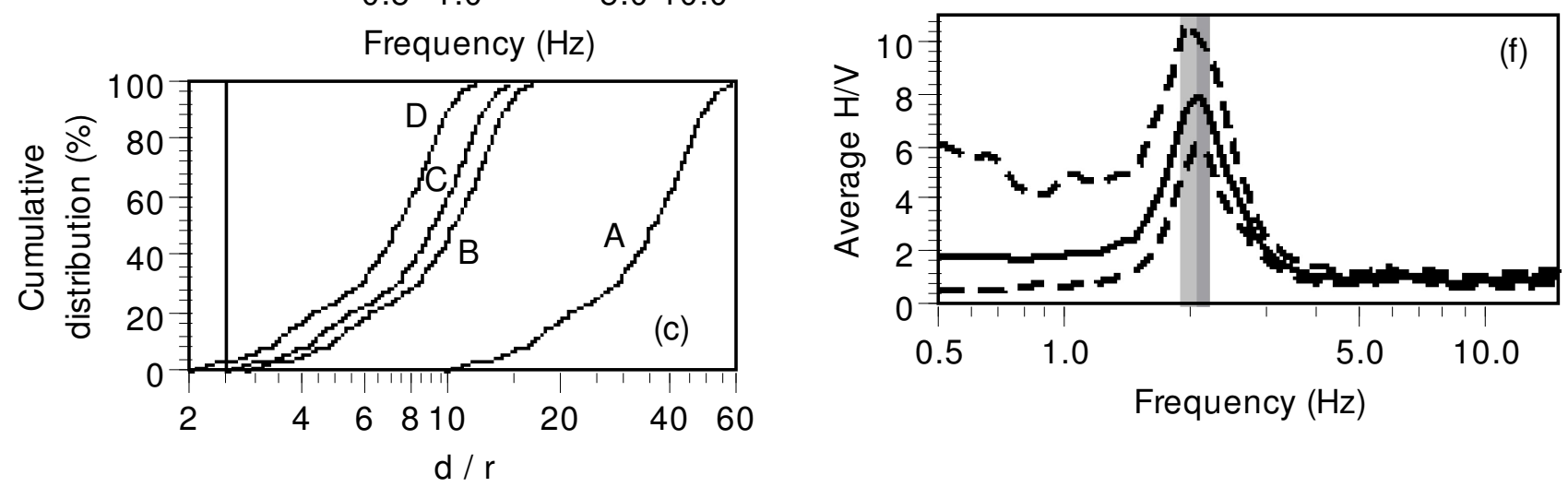

Figure 4: Simulated wavefield: (a) Spatial distribution of sources (black dots) and receivers (gray dots). (b) Source Fourier amplitude spectrum. (c) Distribution of sources versus the ratio of distance to array (d) and its radius (r). (d) and (e) Fourier spectra recorded at the receivers of the arrays on the vertical and east components. The plain line is the average and the dashed lines are located at one standard deviation. (f) Average $\mathrm{H} / \mathrm{V}$ ratio observed for the stations of the arrays A, B, C and D. Grey bands indicate the average and standard deviation of the frequency peak values observed for each individual time window. 

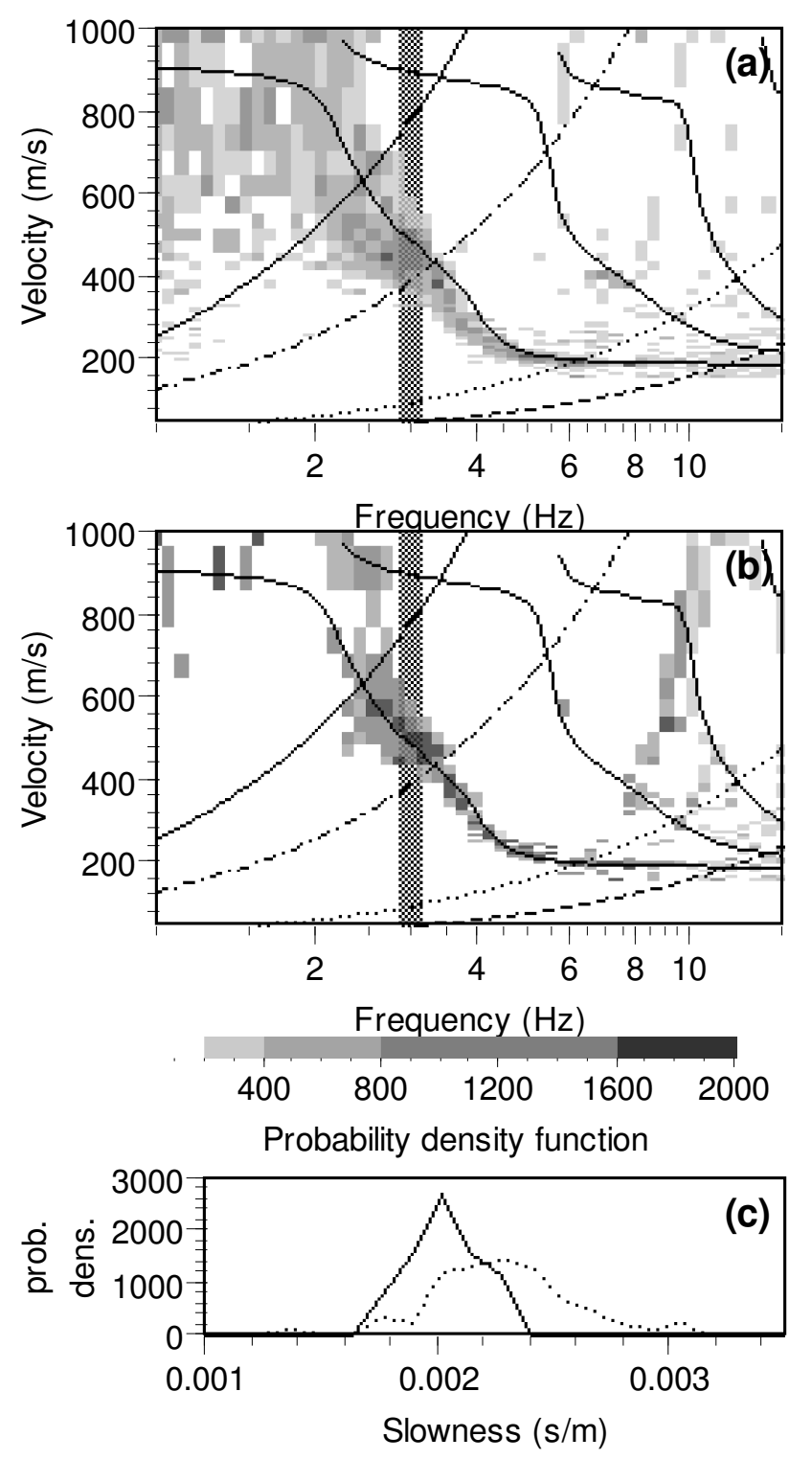

Figure 5: Frequency-wavenumber analysis for array C, influence of the time window length. (a) Histograms of velocities with a beam power peak obtained for time windows of 10 cycles. For each plot, the thin lines are the theoretical dispersion curves for the synthetic model (first four modal curves of Figure 3). The four exponential curves represent constant wavenumber values: $k_{\min } / 2$ (continuous line), $k_{\min }$ (dot-dash line), $k_{\max } / 2$ (dots) and $k_{\max }$ (dashed line). (b) Same processing for time windows of 50 cycles. (c) Cross sections at $3 \mathrm{~Hz}$, through the histograms of Figure (a) and (b), shown by dotted and plain lines, respectively. Horizontal axis is in slowness (see text) 

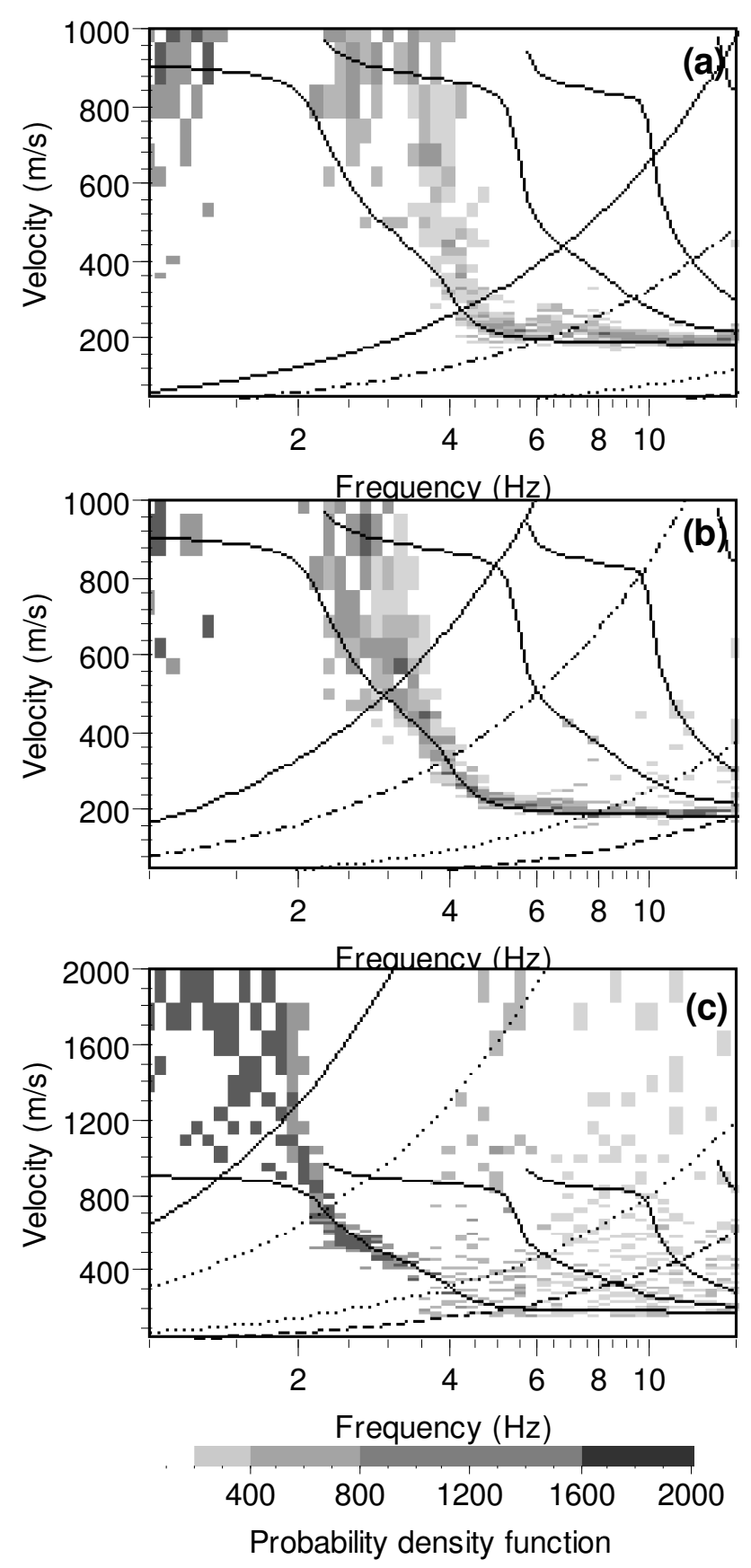

Figure 6: Frequency-wavenumber method applied to arrays A (a), B (b) and D (c) with time windows of 50 cycles as in Figure 5(b). The histograms and the curves are of the same type as in Figure 5. 

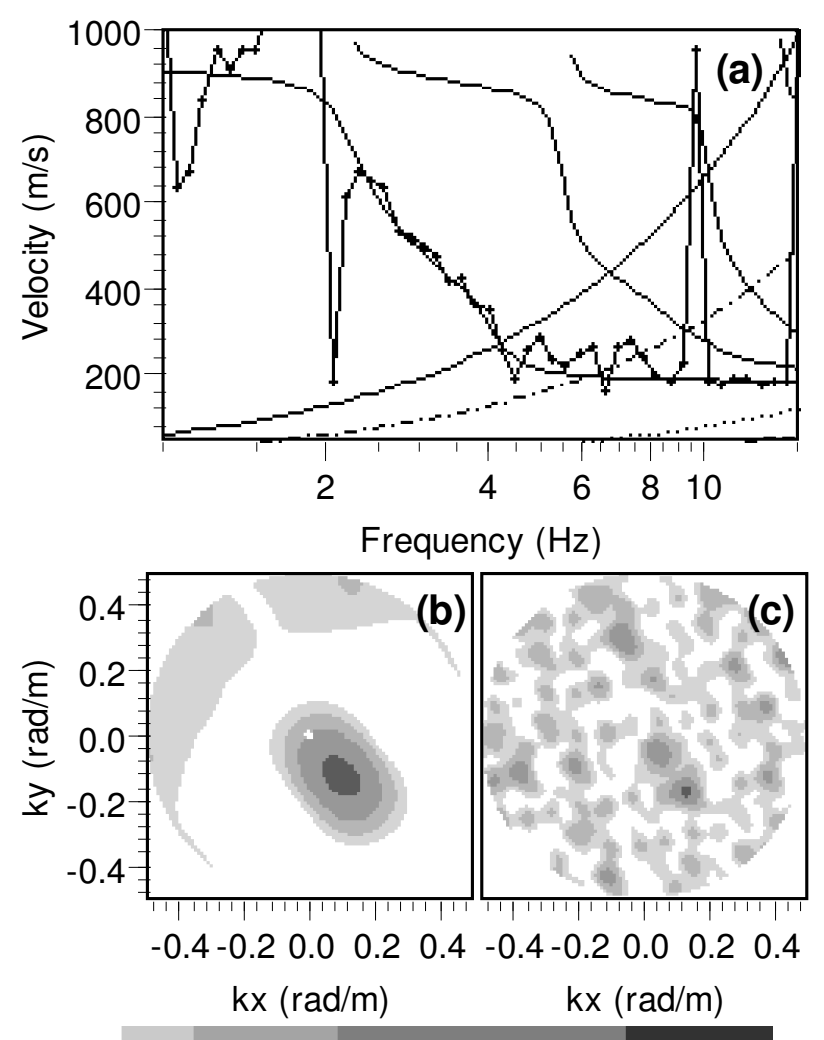

2.0000e-04 4.0000e-04 6.0000e-04 8.0000e-04 Array response

Figure 7: Wavefield composed of a single far source: (a) Frequency-wavenumber analysis for array A. The black dots show the dispersion curve obtained from the frequency-wavenumber method. The other curves are similar to the ones in Figure 5. (b) and (c) Array responses calculated at $6.5 \mathrm{~Hz}$ for arrays $\mathrm{A}$ and $\mathrm{B}$, respectively. 

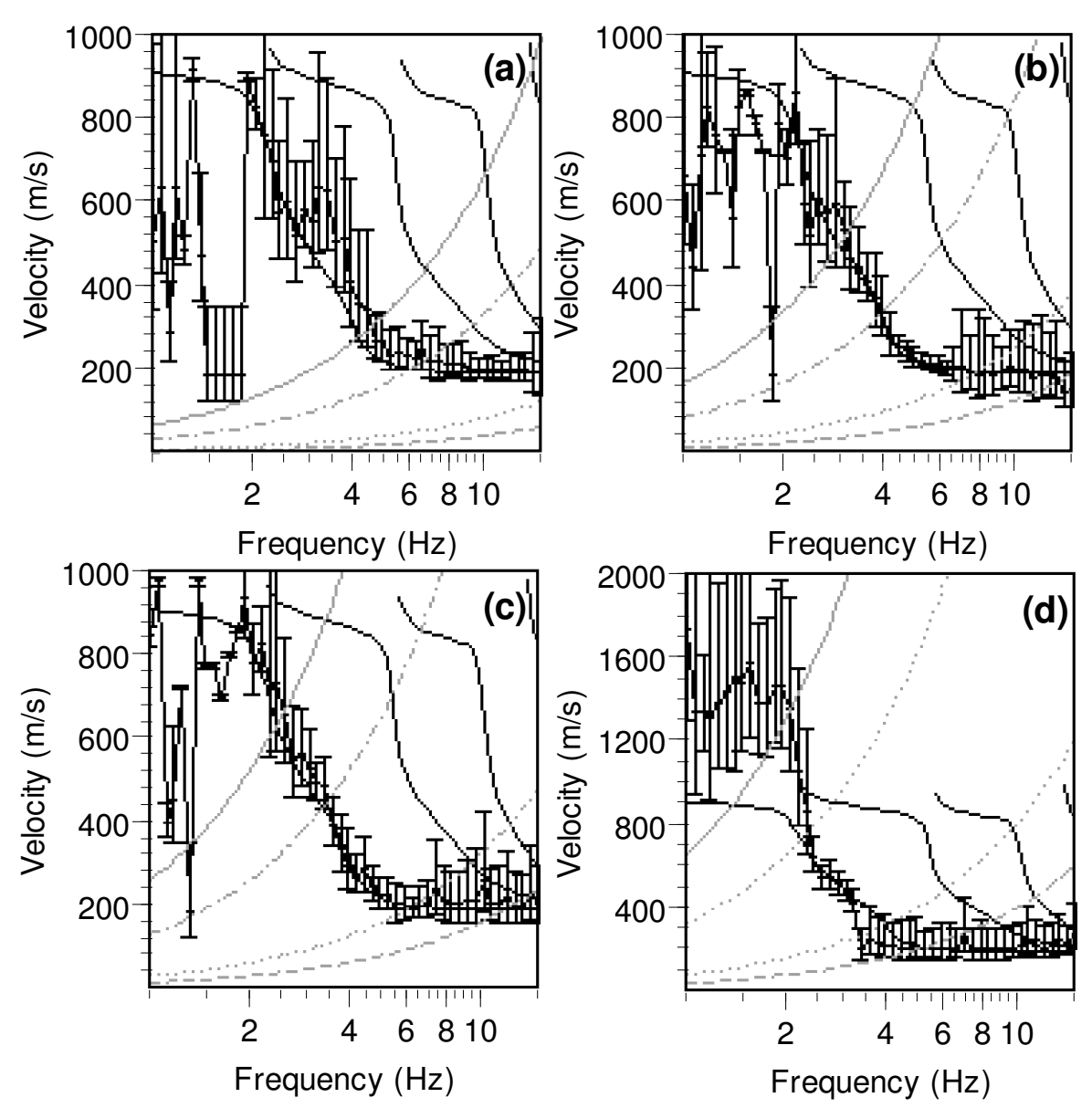

Figure 8: Dispersion curves derived from the high resolution frequency-wavenumber method applied to arrays A (a), B (b), C (c) and D (d). The black lines with dots and error bars, obtained from computations, are compared to the theoretical dispersion curves (thin plain lines). The gray exponential curves are similar to the ones in Figure 5. 

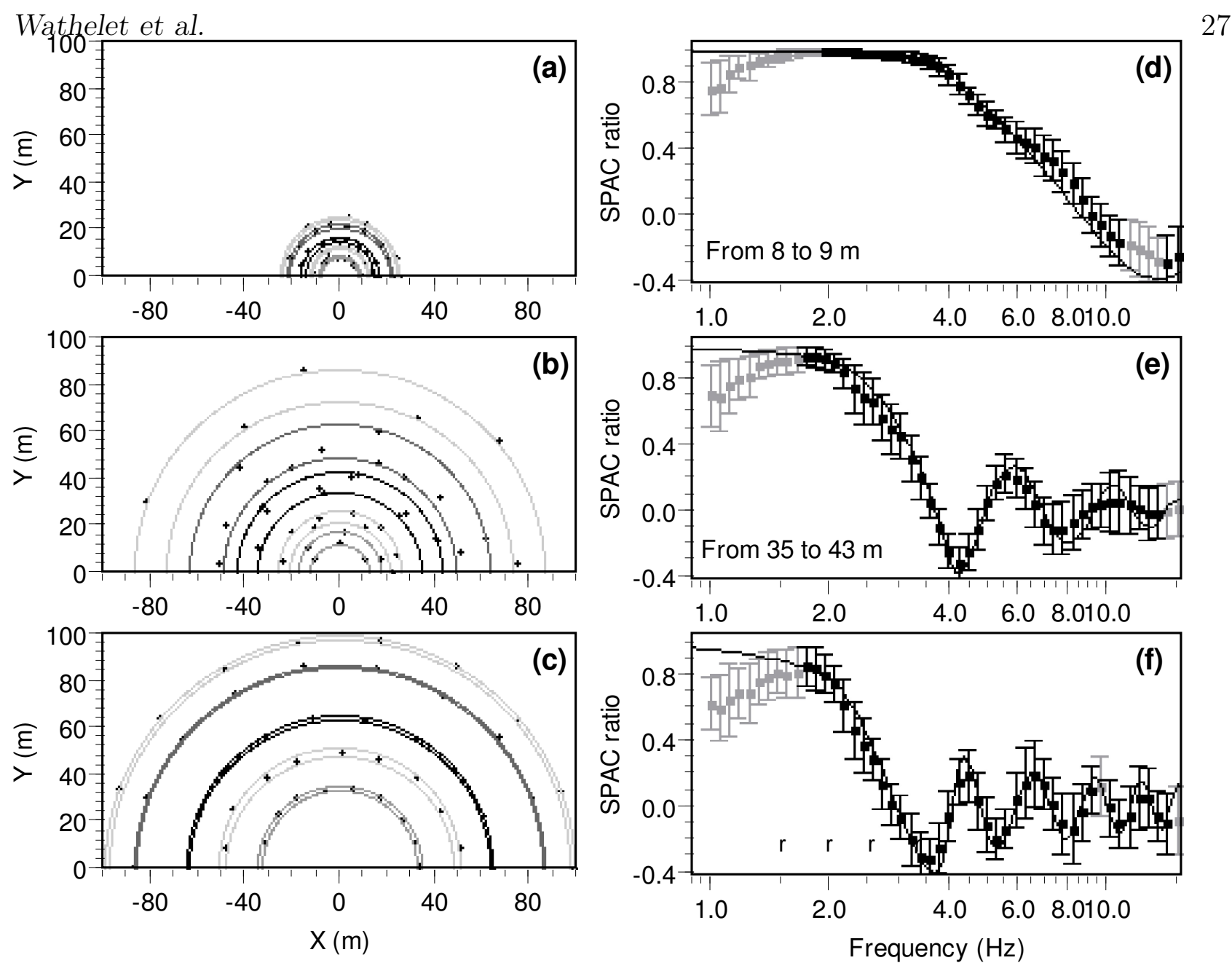

Figure 9: (a), (b) and (c) Azimuth-inter-distance plots for arrays A,B and C, respectively: each dot represents one couple of stations. The pairs of gray circles show the limits of the chosen rings. (d), (e) and (f) Examples of auto-correlation curves obtained for arrays A, B and C, respectively. The black dots and error bars are the samples selected according to criteria of Figure 10. 

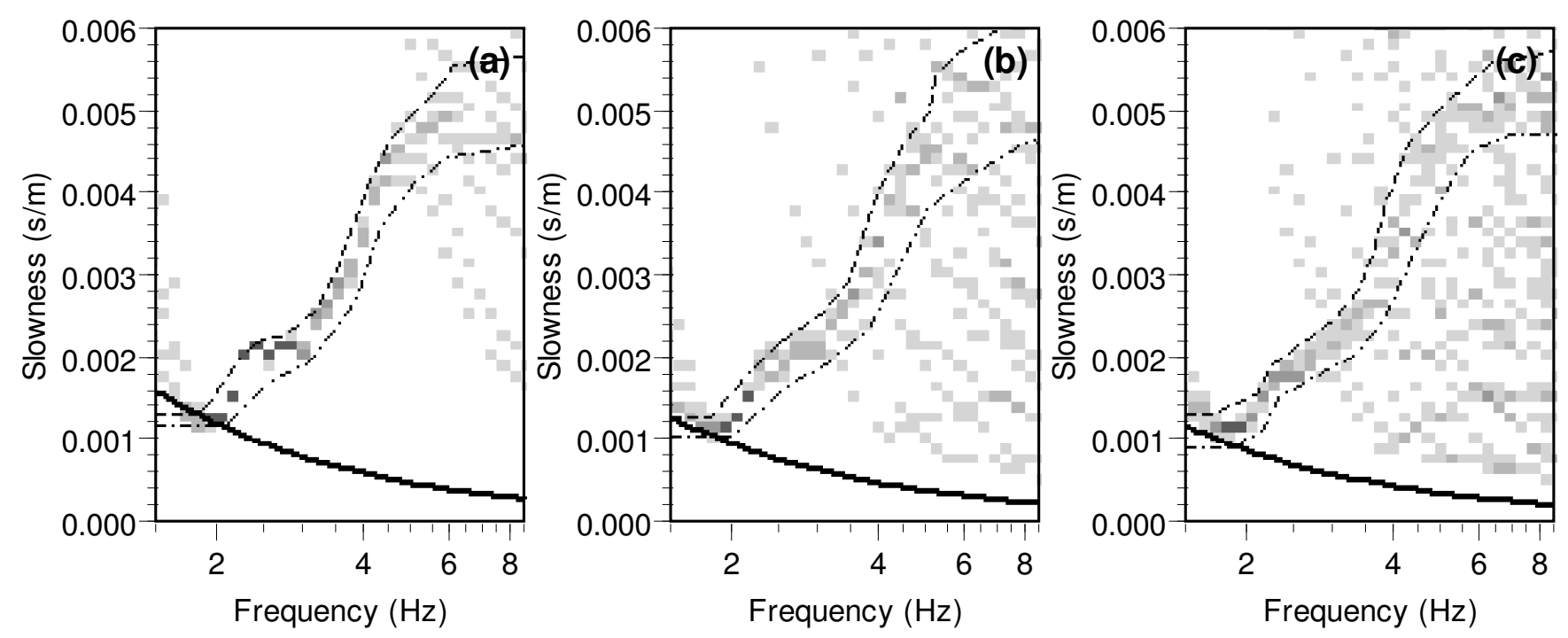

0 2 3

Number of rings with a solution inside cell

Figure 10: (a) to (c) Analysis of the autocorrelation curves obtained for arrays A to C, respectively: grids in frequency-slowness domain representing the density of dispersion curve solutions. The plain lines are the wave number limits deduced from the solution density grids. The dashed and the dot-dashed curves are the limits of the apparent dispersion curve or the limits of the area with a high density of solutions. 

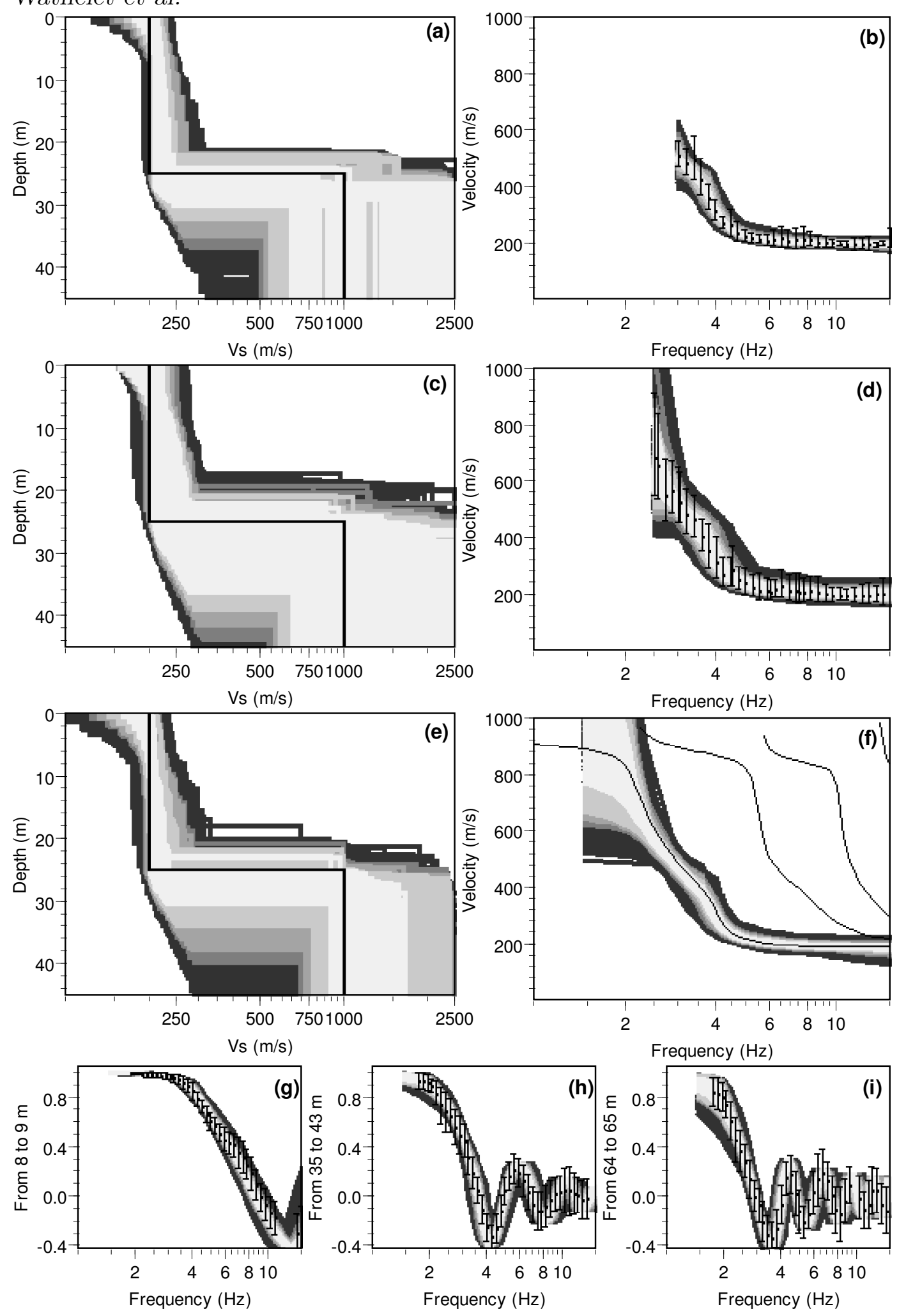

$$
\begin{array}{llll}
0.3 & 0.4 & 0.5 & 0.6 \\
& \text { Misfit value } &
\end{array}
$$

Figure 11: Results of dispersion curve inversion obtained with the three signal processing methods: frequency-wavenumber ( Figure (a) and (b) ), high resolution method ( Figure (c) and (d) ) and spatial auto-correlation ( Figure (e) to (i) ). (a), (c) and (e) $V_{s}$ models and (b), (d), and (f) the corresponding dispersion curves. The dots and error bars represent the experimental dispersion or spatial auto-correlation curves deduced from the processing of arrays A to C. The black lines of Figure (a) and (c), (e) are the velocity profiles of the synthetic model. In Figure (g) to (i), only three of the 15 auto-correlation curves are shown. 